\title{
An Optimized Cosmetic Screening Assay for Ultraviolet B (UVB) Protec- tive Property of Natural Products
}

Camille Keisha Mahendra ${ }^{1}$, Loh Teng-Hern Tan ${ }^{1,2,3}$, Wei Hsum Yap ${ }^{4}$, Chim Kei Chan ${ }^{5}$, Priyia Pusparajah ${ }^{6}$, Bey Hing Goh ${ }^{1,3,7,8^{*}}$
${ }^{1}$ Biofunctional Molecule Exploratory Research Group (BMEX), School of Pharmacy, Monash University Malaysia, 47500
Bandar Sunway, Selangor Darul Ehsan, Malaysia.
${ }^{2}$ Institute of Biomedical and Pharmaceutical Sciences, Guangdong University of Technology,
Guangzhou 510006, PR China.
${ }^{3}$ Novel Bacteria and Drug Discovery Research Group (NBDD), Microbiome and Bioresource Research Strength, Jeffrey Cheah School of Medicine and Health Sciences, Monash University, Bandar Sunway, Malaysia.
${ }^{4}$ School of Biosciences, Taylor's University, Subang Jaya, Selangor, Malaysia
${ }^{5}$ de Duve Institute, Avenue Hippocrate 74, 1200 Brussels, Belgium.
${ }^{6}$ Medical Health and Translational Research Group (MHTR), Jeffrey Cheah School of Medicine and Health Sciences, Monash University Malaysia, 47500 Bandar Sunway, Selangor Darul Ehsan, Malaysia.
${ }^{7}$ Center of Health Outcomes Research and Therapeutic Safety (Cohorts), School of Pharmaceutical Sciences, University of Phayao, Phayao, Thailand.
${ }^{8}$ Institute of Pharmaceutical Science, University of Veterinary and Animal Science Lahore, Pakistan.

\begin{abstract}
In an era where everyone is seeking everlasting youth, preventing photodamage by ultraviolet radiation (UVR) from the sun is of key interest to the cosmetic industry. Inhibiting the production of UVR-mediated reactive oxygen species (ROS) is often the targeted function of cosmetic products, and a suitably reliable model is needed to test this. Hence, the current protocol was designed to effectively analyze the ultraviolet (UV) protective effect of bioactive natural product compounds against photodamage caused by UVR, specifically UVB radiation. Analysis of bioactive compounds that were previously reported to have UV protective properties using this model yielded data that showed the reproducibility and reliability of this model in determining UVB protective properties of the bioactive natural product compounds of interest. Thus, this setup model can be used for future endeavors such as the study of UVB protective properties of other natural products or as a basis of a model for UVA and UVC protective designs.
\end{abstract}

Keywords: Ultraviolet radiation (UVR); cosmetic; reactive oxygen species (ROS); broadband ultraviolet B (BBUVB); natural products; U.S. Food and Drug Administration (FDA); International Organization for Standardization (ISO)

Received: $28^{\text {th }}$ February 2019

Accepted: $28^{\text {th }}$ March 2019

Published Online: $15^{\text {th }}$ April 2019

*Correspondence to: Bey-Hing Goh, School of Pharmacy, Monash University Malaysia, 47500 Bandar Sunway, Selangor Darul Ehsan, Malaysia; goh.bey.hing@ monash.edu.

Citation: Mahendra CK, Tan LTH, Yap WH, et al. An Optimized Cosmetic Screening Assay for Ultraviolet B (UVB) Protective Property of Natural Products. Prog Drug Discov Biomed Sci 2019; 2(1): a0000021

\section{Introduction}

There are three types of UVR produced by the sun that are present in our atmosphere - UVA which has the longest wavelength (320-400nm) and about $95 \%$ penetration level through the ozone, followed by UVB (290-320nm) with around 5\% penetration level and finally UVC (200$290 \mathrm{~nm}$ ) which is absorbed by the upper layer of the stratosphere $^{[1]}$. Only UVA and UVB get absorbed by the skin - UVA, which has a longer wavelength is able to penetrate into our dermis layer, while UVB which has a shorter wavelength gets absorbed mainly in the epidermis ${ }^{[2]}$.
When the skin is exposed to UVA and UVB, a cascade of events is initiated. UVA and UVB irradiation elevate the level of reactive oxygen species (ROS) in the skin, causing indirect DNA damage ${ }^{[3]}$. UVB also causes the production of reactive nitrogen species (RNS) by activating nitric oxide synthase (iNOS) which generates nitric oxide $(\mathrm{NO})$ and nitrotyrosine $\mathrm{e}^{[4]}$. Both ROS and RNS depletes the skin's antioxidant defense system which brings about oxidative and nitrosative stress in the cells. Under stress, the skin cells start initiating pro-inflammatory cytokines and other inflammatory mediators that signal the start of inflammation ${ }^{[5]}$. The formation of melanin is also activated indirectly through the upregulation of tyrosinase and tyrosinase -related protein 1 (TRP-1) 
as a protective mechanism against DNA damage ${ }^{[6]}$. Besides that, the skin's connective tissue also experience damage when exposed to UVR as neutrophils begin to secrete neutrophil elastase that not only cleaves extracellular matrices but also activates matrix metalloproteases (MMP) which assists with the degradation ${ }^{[7]}$. UVB has been demonstrated to be capable of causing direct damage to DNA as it is able to form cyclobutene pyrimidine dimers (CPD) and pyrimidine-pyrimidone (6-4) photoproduct in $\mathrm{DNA}^{[8]}$. As a result of these reactions, under prolonged irradiation, the skin would experience photoaging which is characterized by pigment irregularities, wrinkles, sagging skin and lack of resilience ${ }^{[9]}$.

Bioactive compounds from natural products have proven to have great potential as a source of compounds that could contribute significantly in the fields of medicine $^{[10,11]}$ and cosmetic studies ${ }^{[12-15]}$. In cosmetics, many bioactive compounds obtained from natural products, such as antioxidants, have been used to inhibit ROS induced by UVR. This in turn reduces the oxidative stress in the skin, thus slowing down photoaging ${ }^{[16]}$. In a study done by Pérez-Sánchez, Barrajón-Catalán ${ }^{[17]}$, it was suggested that lemon balm was able to protect human keratinocytes against the detrimental effects of UVB due to its a high content of antioxidants, such as rosmarinic acid, which inhibits the production of ROS. Given that this creates a need for a reliable and cost effective way to screen natural compounds for the photoprotective properties, we have devised and optimized a reliable model to screen for UV protective properties in natural products against the detrimental effect of UVB. To ensure the reliability of this model, bioactive compounds, obtained from natural products that were previously reported to have photoprotective properties were used. This set up model was also made in alignment with the requirements of U.S. Food and Drug Administration (FDA) and International Organization for Standardization (ISO) regulations on UVB exposure in which a broadband UVB (BBUVB) lamp was used to mimic the UVB wavelength that are present in the atmosphere $^{[18,19]}$. The overview of the setup model can be seen in Figure 1.

\section{Method details}

Optimization of UVB exposure

Materials

Basic consumables such as cell culture flasks, 96 well plates, $15 \mathrm{ml}$ or $50 \mathrm{ml}$ Falcon tubes, serological pipettes

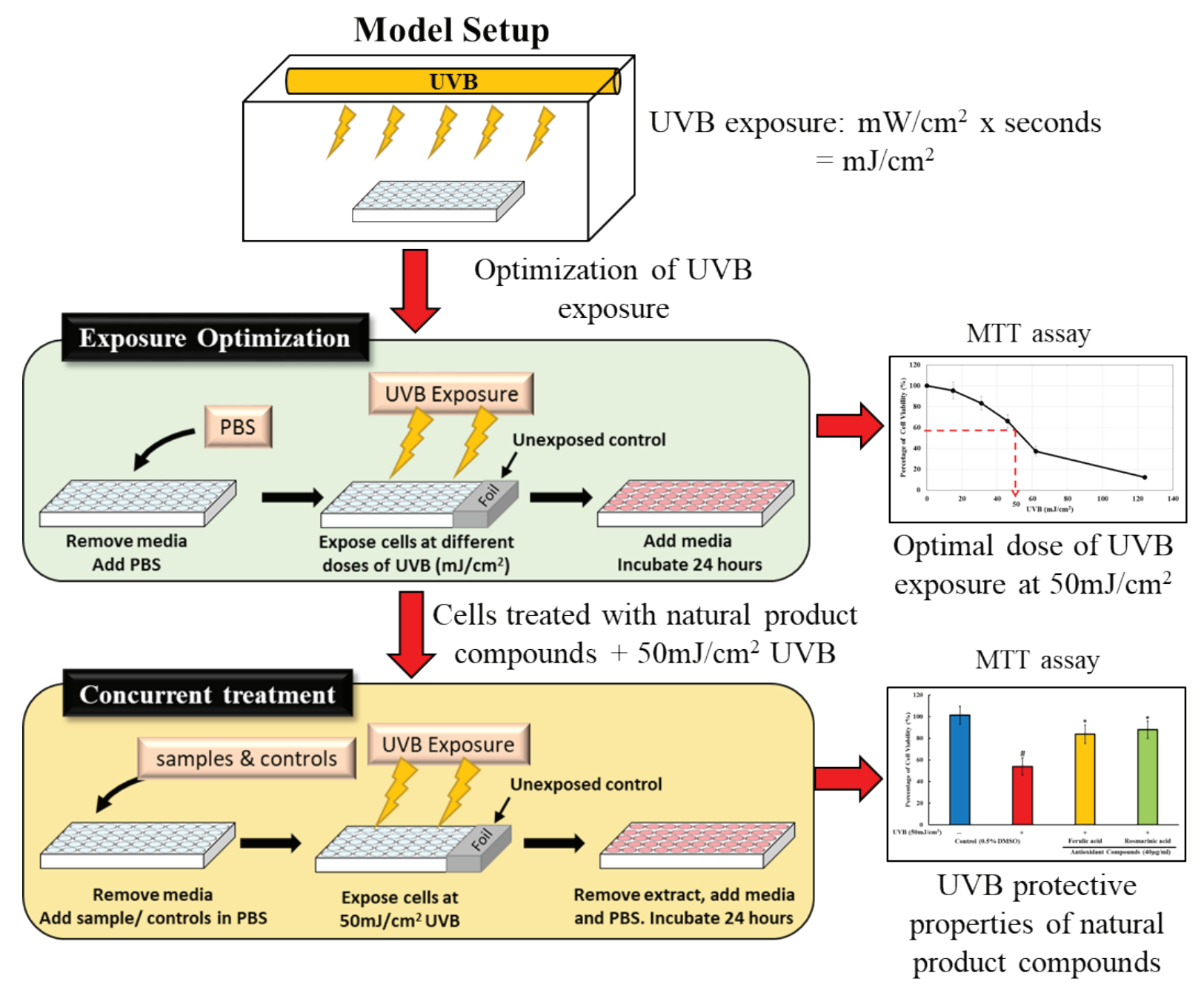

Figure 1. Optimized setup model to determine UVB protective properties of natural products. Optimal dose of UVB exposure were firstly determined via measuring the cell viability of HaCaT cells that were irradiated with different doses of UVB. After optimal UVB dose was ascertained, bioactive compounds from natural products were screened for UVB protective properties by treating the cells in concurrent with UVB irradiation. Cell viability were determined using MTT assay. 
and pipette tips.

- Well-equipped tissue culture facilities that provides $\mathrm{CO}_{2}$ incubator, centrifudge, biosafety cabinet, microplate reader and inverted bright field microscope.

- BBUVB lamp: Philip UVB Broadband TL 20W/12 phototherapy lamp

- UV Meter: Lutron UV Light Meter UV-340A

- Immortalized human keratinocytes (HaCaT) cell line

- Cell culture medium: 1x high-glucose Dulbecco Modified Eagle Medium (DMEM) GlutaMAX without HEPES (Gibco, USA) supplemented with 1.0\% of 100x Antibiotic-antimycotic $(100 \mathrm{U} / \mathrm{ml}$ penicillin, $100 \mu \mathrm{g} / \mathrm{ml}$ streptomycin and $25 \mu \mathrm{g} / \mathrm{ml}$ amphotericin B) (Gibco, USA) and 10.0\% fetal bovine serum (Gibco, USA).

- $\quad$ 1x Phosphate buffered saline (PBS) pH7.4

- $\quad 1 x$ Tryple Express (Gibco, USA)

- $0.4 \%$ Trypan blue stain

- Aluminum foil

Procedure

The HaCaT cells at log-phase of growth, which is approximately $70-80 \%$ confluence was used during all treatments. For all treatment, the cells were seeded at a cell density of $1 \times 10^{5}$ cells $/ \mathrm{ml}$ before incubating for 24 hours at $37^{\circ} \mathrm{C}$ in $5 \% \mathrm{CO}_{2}$ atmosphere to allow adherence ${ }^{[20]}$. The cells were irradiated with Philip UVB Broadband TL 20W/12 phototherapy lamp (Philip, Netherlands) which emits UV radiation within the range of $290-315 \mathrm{~nm}$. The emission energy of the lamp was measured using UV Light Meter UV-340A (Lutron, USA) and the cells were irradiated at 0, 15, 31, 46, 62 and $124 \mathrm{~mJ} / \mathrm{cm}^{2}$ UVB. Cell viability was determined using MTT assay and percentage of cell viability were normalized against the viability of unexposed cells (unexposed controls) as depicted in Figure 1.

(Note: It is recommended that the cells should be at a passage number that is lower than 30 as the constant changing of media and PBS in this protocol could stress the cells. Older cells may detach easier from the 96 well plate during the change of media and hence causes variation in its percentage of cell viability.)

1. Remove the media from each well and add in $50 \mu 1$ of PBS to form a thin layer of solution above the cells.

2. Cover the unexposed control cells with aluminum foil to inhibit the penetration of UVB radiations onto the control cells.

3. Irradiate the cells with $0,15,31,46,62$ and $124 \mathrm{~mJ} /$ $\mathrm{cm}^{2}$ UVB.

4. After irradiation, add $150 \mu 1$ of media into the wells to make up to a total volume of $200 \mu \mathrm{l}$ and incubate the cells at $37^{\circ} \mathrm{C}$ with $5 \% \mathrm{CO}^{2}$ for 24 hours.

5. Proceed with MTT assay after the incubation period.
(Note:Before irradiation, the cover of the plate was removed to avoid any absorption of UVB by the plastic cover.)

Concurrent Treatment of HaCaT cells with compounds and UVB irradiation

Materials

- Basic consumables such as cell culture flasks, 96 well plates, $15 \mathrm{ml}$ or $50 \mathrm{ml}$ Falcon tubes, serological pipettes and pipette tips.

- Well-equipped tissue culture facilities that provides $\mathrm{CO}_{2}$ incubator, centrifuge, biosafety cabinet, microplate reader and inverted bright field microscope.

- BBUVB lamp: Philip UVB Broadband TL 20W/12 phototherapy lamp

- UV Meter: Lutron UV Light Meter UV-340A

- Immortalized human keratinocytes (HaCaT) cell line

- Cell culture medium: 1x high-glucose Dulbecco Modified Eagle Medium (DMEM) GlutaMAX without HEPES (Gibco, USA) supplemented with $1.0 \%$ of $100 \mathrm{x}$ Antibiotic-antimycotic $(100 \mathrm{U} / \mathrm{ml}$ penicillin, $100 \mu \mathrm{g} / \mathrm{ml}$ streptomycin and $25 \mu \mathrm{g} / \mathrm{ml}$ amphotericin B) (Gibco, USA) and 10.0\% fetal bovine serum (Gibco, USA).

- $\quad$ 1x Phosphate buffered saline (PBS) pH7.4

- $\quad$ 1x Tryple Express (Gibco, USA)

- $\quad 0.4 \%$ Trypan blue stain

- $\quad$ Aluminum foil

- $\quad$ Sterile dimethyl sulfoxide (DMSO)

- Bioactive compounds prepared in 100\% DMSO: ferulic acid and rosmarinic acid

Procedure

To determine the UVB protective properties of natural compounds, HaCaT cells were concurrently treated with either rosmarinic or ferulic acid $(40 \mu \mathrm{g} / \mathrm{ml})$, which are naturally occurring bioactive compounds found in various natural products, and irradiated with $50 \mathrm{~mJ} / \mathrm{cm}^{2}$ $\mathrm{UVB}^{[21,22]}$. Both compounds were previously reported to have UV protective properties when tested against UVB on $\mathrm{HaCaT}$ cells through attenuation of apoptosis regulatory genes and proteins, reduction of CPD formation, decrease secretion of inflammatory cytokines and reduction of ROS production ${ }^{[23,24]}$. Hence, both compounds make suitable candidates to assess the current model setup as shown in Figure 1. Percentage of cell viability were analyzed via the MTT assay and data obtained were normalized against the viability of unexposed cells (unexposed controls).

1. Mix the compounds with PBS to give a total concentration of $40 \mu \mathrm{g} / \mathrm{ml}$ of the compounds in $0.5 \%$ DMSO. (For the negative control, cells should only be treated with a concentration of $0.5 \%$ DMSO 
in PBS without the presence of any bioactive compounds)

2. Remove the media from each well and add $50 \mu$ of the mixed solution into its designated well.

3. Cover the unexposed control cells with aluminum foil.

4. Irradiate the plate with $50 \mathrm{~mJ} / \mathrm{cm}^{2} \mathrm{UVB}$.

5. Remove the mixed solution and replace with $150 \mu$ l of media and $50 \mu \mathrm{l}$ of PBS to make up to a volume of $200 \mu 1$.

6. Incubate the plate for 24 hours at $37^{\circ} \mathrm{C}$ with $5 \% \mathrm{CO}_{2}$.

7. Proceed with MTT assay after the 24 hour incubation.

\section{MTT assay}

Materials

- $\quad$ Dimethyl sulfoxide (DMSO)

- 3-(4,5-Dimethylthiazol-2-Y1)-2,5-Diphenyltetrazolium Bromide (MTT) reagent

\section{Procedure}

Cell viability was assessed using 3-(4,5-Dimethylthiazol2-Y1)-2,5-Diphenyltetrazolium Bromide (MTT) assay as described by ${ }^{[2]}$ with slight modifications.

1. Add $20 \mu 1$ of MTT solution into each well.

2. Incubate the plate for 2 hours to allow the formation of formazan crystals

3. Aspirate the solution and add in $100 \mu 1$ of DMSO into each well to solubilize the formazan crystal

4. Measure the absorbance at 570nm using a microplate reader.

5. Calculate the percentage of cell viability after normalizing all samples and negative control against the unexposed control cells.

(Note: All the data obtained were analyzed with one-way analysis of variance (ANOVA) and Tukey HSD post-hoc tests using Statistical Package for Social Science (SPSS) version 24.0. Significance of data was determined set at $\mathrm{p}<0.05$.)

\section{Methods Validation}

\section{Determination of optimal UVB exposure}

To determine the optimal dose of UVB exposure, HaCaT cells were irradiated with $0,15,31,46,62$ and $124 \mathrm{~mJ} / \mathrm{cm}^{2}$ UVB. The data obtained as shown in Figure 2 and microscopic view of the cells in Figure 3 showed a decrease in cell viability as the UVB exposure increases. Cells that were exposed to UVB irradiation were normalized against the unexposed control cells, which was assumed to be at $100 \%$ viability. A significant decrease in cell viability can be seen when the cells were exposed to $31 \mathrm{~mJ} / \mathrm{cm}^{2} \mathrm{UVB}$, where $83.29 \pm 6.55 \%$ of the cells remained viable after the exposure. At the dosage of $45 \mathrm{~mJ} / \mathrm{cm}^{2}$, the viability of the cells decreased to $66.04 \pm 6.48 \%$. While cells that were exposed with 62 and $124 \mathrm{~mJ} / \mathrm{cm}^{2}$ UVB retained $37.31 \pm$ $4.75 \%$ and $12.32 \pm 1.96 \%$ viability respectively.

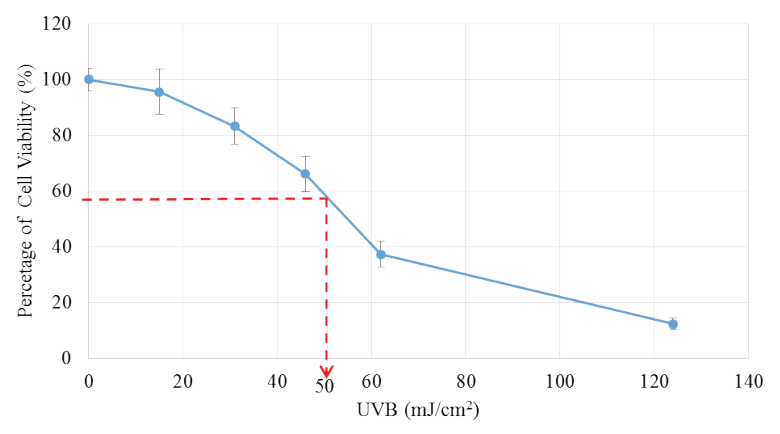

Figure 2. HaCaT cells were subjected to different doses of UVB exposure $\left(\mathrm{mJ} / \mathrm{cm}^{2}\right)$ to determine the optimal dose of UVB exposure. The media from each well were replaced with a thin layer of $\mathrm{PBS}$ and then the cells were exposed to $0,15,31,46,62$ and $124 \mathrm{~mJ} / \mathrm{cm}^{2} \mathrm{UVB}$. The cell viability was then determined through MTT assay after 24 hours incubation. Exposed cells were normalized against unexposed cells (unexposed controls) to obtain the percentage of viable cells. Each data was expressed as mean \pm standard error and $n>3$.
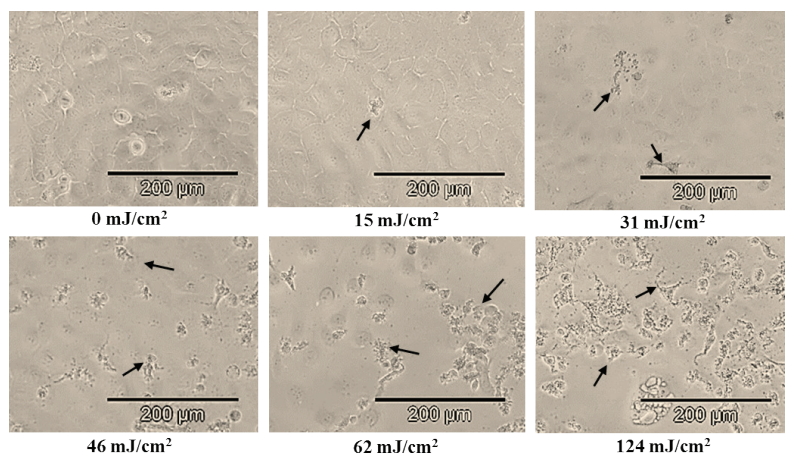

Figure 3. Microscopic view of HaCaT cells after exposure to UVB. The cells were exposed to UVB $(0-124 \mathrm{~mJ} /$ $\mathrm{cm}^{2}$ ) and incubated for 24 hours. The images depict 10x magnification of the cells at each exposure dosage before MTT assay was performed. The arrows indicate UVB-induced dead cells.

UVB protective properties of natural product compounds against UVB

Both ferulic acid and rosmarinic acid, were analyzed for their UV protective effect against UVB. The results obtained (Figure 4) showed that both compounds displayed significant UVB protection on $\mathrm{HaCaT}$ cells during UVB irradiation as compared to the negative control (cells exposed to UVB without the addition of bioactive compounds). The results obtained correlates with other studies that reported UVB photoprotective properties of both ferulic acid and rosmarinic acid ${ }^{[23,26]}$. This shows that the model that was designed is reliable in screening for UVB photoprotective properties of natural products. In the application of cosmetic studies especially for sunscreens, this screening method could prove to be a quick preliminary technique in determining if the added natural product extracts have UV protective properties. This will aid in narrowing down potential plant or other 
biological extracts and thus saving cost in research and development. The presented method can also potentially be developed further to include studies on 3D models of skin tissues to allow for better analysis on the UV protection of developed cosmetics.

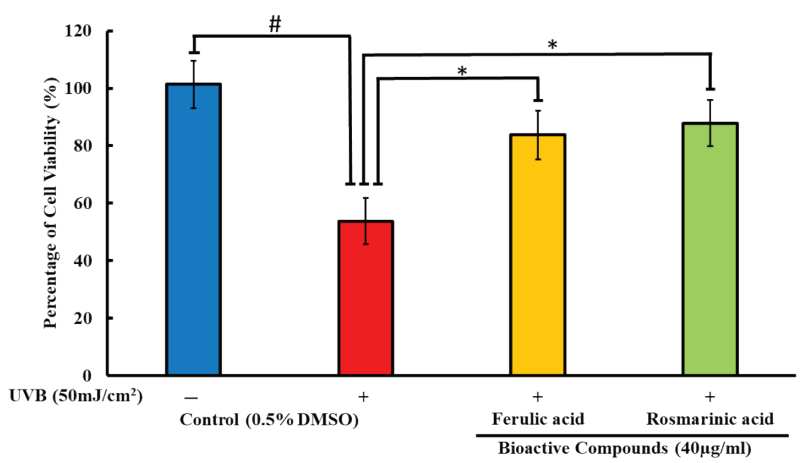

Figure 4. Protective effect of bioactive compounds obtained from natural products against UVB irradiation on $\mathrm{HaCaT}$ cells. HaCaT cells were treated with either ferulic or rosmarinic acid $(40 \mu \mathrm{g} / \mathrm{ml})$ in concurrent with UVB irradiation. Both negative control and unexposed control cells were treated with only $0.5 \%$ DMSO. All cells were irradiated with $50 \mathrm{~mJ} / \mathrm{cm}^{2} \mathrm{UVB}$ except the unexposed control cells. After incubating for 24 hours, MTT assay was done to obtain the cell viability. Irradiated cells were normalized against the viability of unexposed cells to obtain the percentage of cell viability. The viability of negative control cells was compared against unexposed cells while the cells treated with compounds were compared against the negative control. Each data was expressed as mean \pm standard error. (Negative control: $\mathrm{n}>3, \# \mathrm{p} \leq 0.05$ )(Compound: $\mathrm{n}>$ $3, * \mathrm{p} \leq 0.05)$.
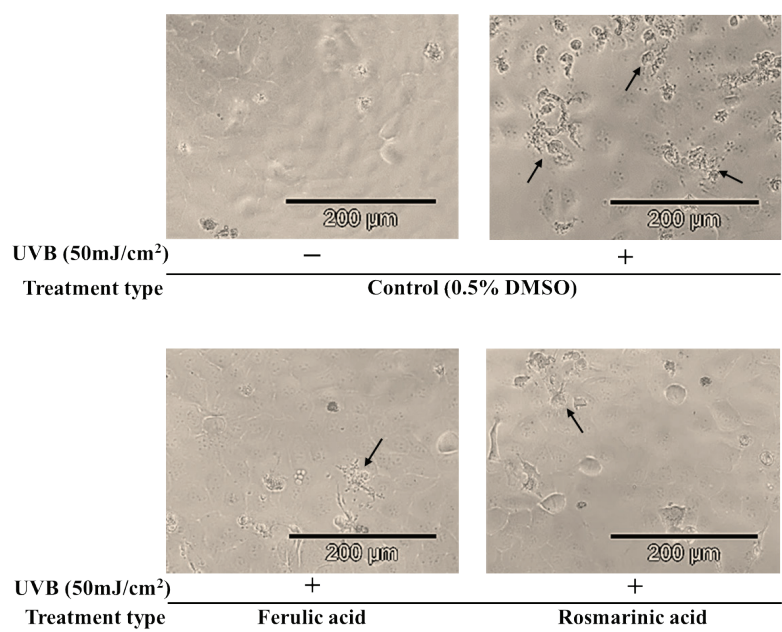

Figure 5. A 10x magnification microscopic view of HaCaT cells that were treated with bioactive compounds and irradiated with UVB. Cells were treated with ferulic or rosmarinic acid $(40 \mu \mathrm{g} / \mathrm{ml})$ and irradiated with $50 \mathrm{~mJ} / \mathrm{cm} 2$ UVB. Both negative control and unexposed control cells were treated with only $0.5 \%$ DMSO prior to irradiation. All cells, except the unexposed control, were irradiated with UVB. The cells were incubated for 24 hours before being treated with MTT assay. The arrows in the figure indicates UVB-induced dead cells. ("+" symbol = exposed cells; " _" symbol $=$ unexposed cells)

\section{Summary}

Overall, the photoprotective properties displayed by ferulic and rosmarinic acid using our set up model were similar to those that were reported by other studies, proving that the model designed is a reliable and replicable method to test for UVB photoprotection properties in natural products. Using this model, other natural products can be easily screened for UVB protective properties, aiding cosmetic companies in the preliminary search for potential UVB protection in natural product extract to include in their formulation. Analysis including 3D skin tissue models can also be developed from this model, allowing further UV protection analysis on the developed product. Finally, this setup model can also be form the basis for development of research models which include screening for protection against UVA and UVC.

\section{Acknowledgements}

This research was financially supported by External Industry Grant (Biotek Abadi - Vote No. GBA-81811A) \& Taylor's University Emerging Grant (TRGS/ ERFS/2/2018/SBS/016).

\section{Conflict of Interest}

The authors declared that there is no conflict of interest.

\section{References}

1. Widel M, Krzywon A, Gajda K, et al., Induction of bystander effects by UVA, UVB, and UVC radiation in human fibroblasts and the implication of reactive oxygen species. Free Radic Biol Med, 2014. 68: 278-287.

2. Berkey C, Biniek K, and Dauskardt RH, Screening sunscreens: protecting the biomechanical barrier function of skin from solar ultraviolet radiation damage. Int J Cosmet Sci, 2017. 39(3): 269-274

3. Kovacs D, Salvatore R, Enrica F, et al., Keratinocyte growth factor down-regulates intracellular ROS production induced by UVB. $J$ Dermatol Sci, 2009. 54(2): 106-113.

4. Filip GA, Postescu ID, Pompei B, et al., Inhibition of UVB-induced skin phototoxicity by a grape seed extract as modulator of nitrosative stress, ERK/NF-kB signaling pathway and apoptosis, in SKH-1 mice. Food Chem Toxicol, 2013. 57: 296-306.

5. Bashir MM, Sharma MR and Werth VP, UVB and pro-inflammatory cytokines synergistically activate TNF- $\alpha$ production in keratinocytes through enhanced gene transcription. J Invest Dermatol, 2009. 129(4): 994-1001.

6. Nylander K, Bourdon JC, Bray SE, et al., Transcriptional activation of tyrosinase and TRP-1 by p53 links UV irradiation to the protective tanning response. J Pathol, 2000. 190(1): 39-46.

7. Imokawa G, Recent advances in characterizing biological mechanisms underlying UV-induced wrinkles: a pivotal role of fibrobrastderived elastase. Arch Dermatol Res, 2008. 300(1): 7-20.

8. You YH, Lee DH, Yoon JH, et al., Cyclobutane Pyrimidine Dimers Are Responsible for the Vast Majority of Mutations Induced by UVB Irradiation in Mammalian Cells. J Biol Chem, 2001. 276(48): 44688-44694.

9. Scharffetter-Kochanek K, Brenneisen P, Wenk J, et al., Photoaging of the skin from phenotype to mechanisms. Exp Gerontol, 2000 35(3): 307-316.

10. Tan LTH, Low LE, Tang SY, et al., A reliable and affordable 3D tumor spheroid model for natural product drug discovery: A case study of curcumin. Prog Drug Discov Biomed Sci, 2019. 2(1).

11. Ma DS, Tan LT-H, Chan K-G, et al., Resveratrol-Potential Antibacterial Agent against Foodborne Pathogens. Front Pharmacol, 2018. 9: 102.

12. Choi SW, Son BW, Son YS, et al., The wound-healing effect of a glycoprotein fraction isolated from aloe vera. Br J Dermatol, 2001. 145(4): 535-545.

13. Ribnicky DM, Kuhn P, Poulev A, et al., Improved absorption and 
bioactivity of active compounds from an anti-diabetic extract of Artemisia dracunculus L. Int J Pharm, 2009. 370(1): 87-92.

14. Tan LTH, Ser, HL, Yin WF, et al., Investigation of Antioxidative and Anticancer Potentials of Streptomyces sp. MUM256 Isolated from Malaysia Mangrove Soil. Front Microbiol, 2015. 6: 1316.

15. Tan LTH, Lee LH, Yin WF, et al., Traditional uses, phytochemistry, and bioactivities of Cananga odorata (ylang-ylang). Evid Based Complement Alternat Med, 2015. 2015.

16. Afaq F and Mukhtar H, Botanical antioxidants in the prevention of photocarcinogenesis and photoaging. Exp Dermatol, 2006. 15(9): 678-684.

17. Pérez-Sánchez A, Barrajón-Catalán E, Herranz-López M, et al., Lemon balm extract (Melissa officinalis, L.) promotes melanogenesis and prevents UVB-induced oxidative stress and DNA damage in a skin cell model. J Dermatol Sci, 2016. 84(2): 169-177.

18. ISO. ISO 24444:2010 Cosmetics - Sun protection test methods - In vivo determination of the sun protection factor (SPF). 2010 [cited 2018; Available from: https://www.iso.org/obp/ui/\#iso:std:iso:24444:ed1:v1:en.

19. FDA. Guidance for Industry Labelling and Effectiveness Testing: Sunscreen Drug Products for Over-The Counter Human Use - Small Entity Compliance Guide. 2012 [cited 2018; Available from: https://www. fda.gov/drugs/guidancecomplianceregulatoryinformation/guidances/ ucm330694.htm.

20. Jang J, Ye BR, Heo SJ, et al., Photo-oxidative stress by ultraviolet$B$ radiation and antioxidative defense of eckstolonol in human keratinocytes. Environ Toxicol Pharmacol, 2012. 34(3): 926-934.

21. Kumar N, and Pruthi V, Potential applications of ferulic acid from natural sources. Biotechnol Rep, 2014. 4: 86-93.

22. Wang H, Provan GJ, and Helliwell K, Determination of rosmarinic acid and caffeic acid in aromatic herbs by HPLC. Food Chemistry, 2004. 87(2): 307-311

23. Vostálová J, Zdařilová $\mathrm{A}$, and Svobodová $\mathrm{A}$, Prunella vulgaris extract and rosmarinic acid prevent UVB-induced DNA damage and oxidative stress in HaCaT keratinocytes. Arch Dermatol Res, 2010. 302(3): 171-181.

24. Lin XF, Min W, and Luo D, Anticarcinogenic effect of ferulic acid on ultraviolet-B irradiated human keratinocyte HaCaT cells. J Med Plant Res, 2010. 4(16): 1686-1694.

25. Goh BH, and Kadir AH, In vitro cytotoxic potential of Swietenia macrophylla King seeds against human carcinoma cell lines. J Med Plant Res, 2011. 5(8): 1395-1404.

26. Ambothi $\mathrm{K}$, and Nagarajan RP, Ferulic acid prevents ultraviolet-B radiation induced oxidative DNA damage in human dermal fibroblasts. Int J Nutr Pharmacol Neurol, Pharmacology, Neurological Diseases, 2014. 4(4): 203-213. 